\title{
AYUDA AL OPERARIO EN LA DISTRIBUCIÓN ÓPTIMA DE CARGA ENTRE EQUIPOS EQUIVALENTES
}

\author{
Carlos Gómez Palacín, César de Prada, José Luis Pitarch \\ Instituto de Procesos Sostenibles (IPS). Dpto. Ingeniería de Sistemas y Automática. \\ Universidad de Valladolid, C/ Real de Burgos s/n, 47011, Valladolid. \\ \{carlos.gomez|prada|jose.pitarch\}@autom.uva.es
}

\section{Resumen}

Este trabajo se centra en la planificación de tareas en una línea de producción semi-continua de una planta alimentaria, también llamada la programación o el "scheduling"de la línea. Más concretamente en la distribución de carga entre equipos similares. Dadas las características especiales del producto tratado, destinado a consumo humano, existen restricciones de calidad (tiempos de procesamiento y almacenado, y temperaturas de esterilización entre otros) más estrictas que en otros tipos de industria.

A diferencia de las herramientas de planificación habituales que se emplean en las tareas de organización general de la planta, el estudio presenta una herramienta enfocada al uso en tiempo real por los operarios de un tramo concreto de las líneas de producción.

Palabras clave: Scheduling, RTO, autoclave.

\section{INTRODUCCIÓN}

Con la creciente competencia entre industrias, un correcto secuenciamiento y una planificiación adecuada de las tareas que pueden acortar los tiempos de producción dejan de ser aspectos secundarios, para pasar a ser clave en la gestión global de las plantas. Las herramientas actuales, aplicando en la mayoría de los casos reglas heurísticas para facilitar la obtención de soluciones, se emplean para la organización semanal del trabajo de las fábricas, repartiendo los trabajos por horas de dedicación entre los distintos equipos y operarios de planta, [3]. Se focalizan, principalmente, en respetar los tiempos de entrega pactados por las capas superiores de la gestión, o en minimizar retrasos que pueden acarrear penalizaciones económicas. Sin embargo, estas herramientas no son útiles en el trabajo diario llevado a cabo por los operarios en la planta, los cuales reciben órdenes de trabajo que deben cumplir sorteando problemas de falta de recursos o breves paradas en las líneas. Este trabajo busca obtener una herramienta para un uso en tiempo real por los operarios, que sea eje- cutada con una alta frecuencia, en comparación a las habituales, y calcule el óptimo de las acciones en un breve horizonte de predicción indicando al trabajador qué acciones acometer en cada momento temporal.

Del mismo modo, la sincronización de distintos procesos interfiere en el consumo de recursos compartidos, léase como tales materias primas, vapor, electricidad, trabajadores, equipos, etcétera, por lo que una planificación óptima no sólo repercute en los tiempos de entrega, sino que puede rebajar los costes asociados estableciendo como meta un uso eficiente de los recursos. Si bien, como ya se indicó, con las herramientas de gestión se priorizan los tiempos de finalización, con una herramienta en tiempo real se puede primar la ejecución rápida en algunos casos con mayor producción, y en momentos más holgados, favorecer el ahorro de algún recurso.

En las plantas industriales actuales es normal encontrarse con líneas de producción semi-continuas, esto es alguno de los procesos requiere un procesamiento por lotes, mientras que el resto de la línea produce de manera continua, ya sea un llenado de envases, un tratamiento térmico, un empaquetado de producto final, un almacenado, etcétera. En muchos casos, estos procesamientos requieren de la intervención de algún operario, lo que implica además que no es posible realizar un número indeterminado de estos lotes de manera simultánea, si no que viene definido por la disposición de trabajadores en el momento necesario. En estos casos no sólo hay que tener en cuenta el número de operarios de los que se dispone, sino las tareas que se asignan a cada uno; esto es, una misma tarea puede ejecutarse más rápido si dos operarios la realizan a la vez dos veces seguidas, que si los dos la realizan de manera paralela en equipos distintos, o viceversa.

Este trabajo busca mejorar las órdendes de trabajo dadas por herramientas a más alto nivel, que planifican días o semanas de producción asegurando factibilidad, pero que no pueden asegurar optimalidad a bajo nivel, [1] [2]. El enfoque seleccionado pretende optimizar la agrupación por lotes y la asignación a equipos de los lotes creados, 
cuestiones que no se pueden tener en cuenta cuando se está resolviendo la planificación global de la planta. De esta manera se podrá conseguir una herramienta para que pueda ser usada en tiempo real. Dadas las limitaciones temporales de los algoritmos de resolución mixto-entero no lineales actuales, se opta por implementar el problema como mixto-entero.

El resto del artículo se organiza de la siguiente manera: en la siguiente sección se describe brevemente el sistema a tratar. En la sección 3 se analiza el modelo matemático planteado para abordar el problema. Después, en la sección 4, se exponen los resultados obtenidos. Por último, se trata el trabajo futuro que se está realizando, así como se redactan las conclusiones obtenidas.

\section{CASO PRÁCTICO}

Las soluciones obtenidas en este trabajo se van a implementar en el caso concreto de una planta conservera del noroeste español. En concreto, en las líneas de enlatado de túnidos de ésta. Tras distintas etapas continuas de llenado de los envases y sellado de los mismos, las latas deben ser esterilizadas en un proceso por lotes. Este proceso de esterilización consiste en un tratamiento térmico en autoclaves industriales, donde se introducen las latas en gran número un tiempo y a una temperatura determinados por los tipos de contenido y continente de la conserva, lo que conlleva que todos los envases en el mismo lote de esterilización sean similares, sino iguales.

Para manejar los envases de manera más rápida, las líneas de sellado disponen automáticamente las latas en grandes carros metálicos industriales, cuyas paredes son rejas para permitir el flujo del medio usado para calentar a través de ellas. Aunque puede haber varias líneas trabajando a la vez, cada una lo hace con un producto (conserva + envase); es decir, cada carro se rellena sólamente de un tipo concreto, aunque varias líneas distintas puedan estar rellenando carros del mismo producto de manera concurrente. Así se puede considerar que cada carro requiere un perfil de esterilización determinado por el tipo de lata que contiene, y se puede hablar de carros iguales, similares o incompatibles.

Aunque tanto el llenado como el vaciado de los carros se realiza de manera automática, el desplazamiento de los carros desde las líneas de llenado hasta los autoclaves, y desde estos hasta las líneas de empaquetado posteriores, se debe hacer de manera manual por un número limitado de operarios. Estos introducen los carros en los autoclaves hasta completar la capacidad, o hasta que se deja de producir un producto; o bien forman una cola de carros iguales a la entrada del autoclave si este está ocupado. Una vez que se ha cerrado el autoclave, se selecciona el programa necesario y se inicia la esterilización. Cuando el autoclave termina, los operarios extraen los carros y los disponen para su vaciado en las líneas de empaquetado de las latas, donde otros operarios devuelven los carros vacíos para que vuelvan a ser utilizados.

Existen diversas restricciones en cuanto al procesamiento térmico del producto. La primera es que este debe realizarse antes de un tiempo máximo desde que la lata ha sido sellada para evitar la aparición de bacterias y aminas dentro de la conserva. Esta restricción depende del tipo de producto, y no del envase o del condimento que se haya añadido, por lo que es un valor fijo. Si este tiempo se sobrepasa el carro no puede seguir tratándose de manera normal; una vez esterilizado se almacena hasta que se realizan pruebas de calidad para asegurar que el retraso no ha afectado a las propiedades del producto.

Otra serie de restricciones se centran en el perfil térmico, el cual debe adecuarse a una curva precalculada para obtener las características óptimas de conservación de nutrientes del alimento, y mejor aspecto visual, cumpliendo a mayores unas restricciones duras de tiempo mínimo a alta temperatura para asegurar que se ha alcanzado un nivel de letalidad para las bacterias que podrían encontrarse en el pescado. Este perfil sí es dependiente del envase y del producto.

El proceso de esterilizado supone un cuello de botella cuando la producción de la planta es elevada. Por ello se ha optado por permitir la esterilización simultánea de carros similares en el mismo autoclave, reduciendo los tiempos de espera. La herramienta desarrollada facilitará la decisión de reparto de los carros en los autoclaves a los operarios reduciendo los tiempos generales del proceso, de esta forma se acelera la circulación de carros, minimizando los tiempos de espera y el número total de carros necesarios. Liberando a su vez problemas de espacio de almacenamiento de los carros esperando a ser sometidos al proceso de esterilización.

Puede verse una representación gráfica de la sección descrita en la figura 1. Donde se observan carros siendo llenados por latas en las líneas de sellado. Después, la zona de autoclaves, con algunos carros esperando a ser introducidos en los esterilizadores. Por último, la zona de empaquetado donde se liberan los carros para volver a las líneas de sellado. 


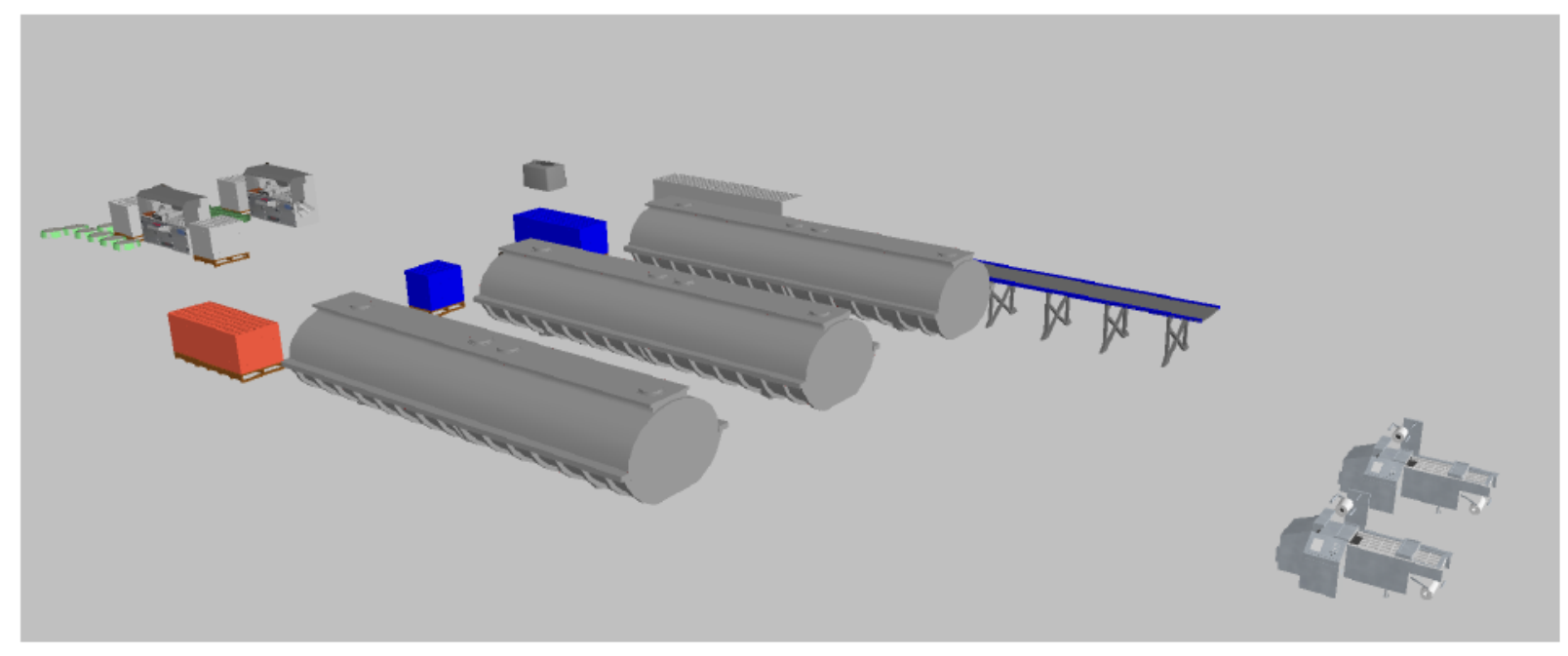

Figura 1: Representación de la planta

\section{FORMULACIÓN MATEMÁTICA}

La herramienta parte de la situación actual de la sección de esterilizado, donde hay una serie de carros ya producidos que tienen que ser sometidos al proceso de esterilizado, y de la planificiación global de la planta, la cual configura unas frecuencias de producción en las líneas de enlatado que permiten calcular instantes de llegada estimados de carros en el futuro inmediato. Se realizan grupos de carros, con los presentes y con los esperados de hasta la capacidad máxima de los autoclaves, y se asignan a los autoclaves disponibles. La herramienta se enfoca en un uso en tiempo real, por lo que el horizonte con el que se trabaja no debe ser demasiado elevado, primando la ejecución rápida del programa.

Se definen tres conjuntos: los carros, $I$; los grupos de carros, $J$; y los autoclaves en sí, $K$. Y las variables binarias que los relacionan: una variable de asignación de carro a grupo, $X_{i, j}\{i \in I, j \in J\}$; una variable de precedencia entre grupos asignados al mismo autoclave, $Y_{j 1, j 2}\{j 1, j 2 \in J\}$ que indica que el grupo $j 1$ se procesa antes que el grupo $j 2$ en el mismo autoclave, sin definir de qué autoclave se trata; y la asignación de los grupos a los autoclaves, $Z_{j, k}\{j \in J, k \in K\}$. Se definen así mismo tres variables reales: el tiempo de inicio del proceso de esterilizado de cada grupo de carros, $S_{j}\{j \in J\}$; la duración del proceso de esterilizado de cada grupo, $P_{j}\{j \in J\}$; y el instante final global, $F$, que no es relativo a ningún conjunto, sino a todos a la vez.

Cada carro tiene fijado un instante de llegada relativo al tiempo actual, $A_{i}$, que corresponde con el ínfimo tiempo de sellado de todas las latas que contiene, de forma que se pueda asegurar que se respeta la restricción de tiempo máximo de espera para el peor caso; y un perfil de esterilizado, con una duración $E_{i}$. Estos datos se extraen directamente de la base de datos de la planta, gracias a los distintos sistemas de tomas de datos que están instalados en la planta.

A continuación se describe el modelo matemático planteado. Primero las ecuaciones de disposición de los carros en grupo, después las restricciones de precedencia de la ejecución de los procesos de esterilizado, seguidas por las relacionadas a los tiempos de proceso. Por último la función de coste.

1. Cada carro sólo puede pertenecer a un grupo de carros.

$$
\forall i \in I \quad \sum_{j \in J} X_{i, j} \leq 1
$$

2. Cada grupo de carros no puede superar la capacidad máxima del equipo.

$$
\forall j \in J \quad \sum_{i \in I} X_{i, j} \leq C
$$

3. Un grupo de carros debe asignarse a un y sólo a un autoclave.

$$
\forall j \in J \quad \sum_{k \in K} Z_{j, k}=1
$$

4. Dos grupos de carros pueden esterilizarse antes o después en un mismo autoclave, pero no los dos a la vez.

$$
\forall j 1, j 2 \in J: j 1 \neq j 2 \quad Y_{j 1, j 2}+Y_{j 2, j 1} \leq 1
$$

5. Si dos grupos de carros están asignados al mismo autoclave, uno debe preceder al otro.

$$
\begin{array}{r}
\forall j 1, j 2 \in J: j 1 \neq j 2, \forall k \in K \\
Y_{j 1, j 2}+Y_{j 2, j 1} \geq Z_{j 1, k}+Z_{j 2, k}-1
\end{array}
$$


6. Cuando dos grupos de carros no están asignados al mismo autoclave, no pueden precederse.

$$
\begin{array}{r}
\forall j 1, j 2 \in J: j 1 \neq j 2, \forall k \in K \\
Y_{j 1, j 2} \leq 1-\left(Z_{j 1, k}-Z_{j 2, k}\right)
\end{array}
$$

7. Cada proceso de esterilizado no puede empezar antes de que todos los carros que contiene hayan sido extraídos de la línea de sellado.

$\forall i \in I, \forall j \in J \quad S_{j} \geq A_{i}-M \cdot\left(1-X_{i, j}\right)$

8. El proceso de esterilizado de un grupo de carros, debe igualar o superar el tiempo mínimo requerido por cada carro que lo compone.

$\forall i \in I, \forall j \in J \quad P_{j} \geq E_{i}-M \cdot\left(1-X_{i, j}\right)$

9. Cada grupo tiene que iniciar su esterilización antes de que pase el tiempo máximo de espera de los carros que lo componen.

$$
\begin{array}{r}
\forall i \in I, \forall j \in J \\
S_{j}-A_{i} \leq W+M \cdot\left(1-X_{i, j}\right)
\end{array}
$$

10. Los carros que no se incluyen en ningún grupo no deben haber llegado con anterioridad a un horizonte de predicción $H$.

$$
\forall i \in I \quad A_{i}+M \cdot \sum_{j \in J} X_{i, j} \geq H
$$

11. Un proceso de esterilización de un autoclave debe esperar a que terminen los previos para iniciarse.

$$
\begin{array}{r}
\forall j 1, j 2 \in J: j 1 \neq j 2 \\
S_{j 1}+P_{j 1} \leq S_{j 2}+M \cdot\left(1-Y_{j 1, j 2}\right)
\end{array}
$$

12. El tiempo final es mayor al tiempo de inicio de cada grupo de carros más su tiempo de procesamiento respectivo.

$$
\forall j \in J \quad F \geq S_{j}+P_{j}
$$

Donde $C$ es el número máximo de carros que pueden ser introducidos en cada autoclave, $W$ es el tiempo máximo de espera de los envases antes de someterse a la esterilización una vez han sido sellados, y $H$ es el horizonte de predicción que asegura que los carros que han sido liberados antes de ese instante se introducen en un autoclave. Así mismo, se define $M$ como un valor mayor al máximo que puedan tomar las restricciones afectadas, para evitar que afecten en los casos que no deban estar activas.
Para acelerar los tiempos de cálculo se han añadido varias restricciones que no afectan al resultado final. La primera que cada grupo de carros debe contener al menos un carro.

$$
\forall j \in J \quad \sum_{i \in I} X_{i, j} \geq 1
$$

Además, se añade una variable de holgura por cada carro, $V_{i}$, que supone la cota superior a la diferencia entre los tiempos de esterilizado necesarios por cada carro y los tiempos de procesamiento del grupo al que pertenecen.

$\forall i \in I, \forall j \in J \quad P_{j} \leq E_{i}+V_{i}+M \cdot\left(1-X_{i, j}\right)$

Por último, se ha creado un orden artificial entre los grupos de carros relativo al orden de los nombres del conjunto $J$ que no afecta al resultado final, puesto que no hay limitaciones en cuanto a la pertenencia de los carros a grupos concretos.

$$
\begin{array}{r}
\forall j 1, j 2 \in J: P O S(j 1) \leq \operatorname{POS}(j 2) \\
S_{j 1} \leq S_{j 2}
\end{array}
$$

Donde $P O S(j) \forall j \in J$ devuelve la posición del elemento $j$ dentro del conjunto ordenado $J$.

$\mathrm{Al}$ minimizar la función de coste, $\mathcal{J}$, se minimizan las diferencias entre los carros asociados en cada grupo, a la vez que se maximizan el número de carros que se esterilizan, de forma que se penaliza realizar operaciones de esterilizado con autoclaves sin completar, a la vez que se busca acabar todos los lotes en el menor tiempo posible.

$$
\mathcal{J}=\alpha \cdot \sum_{i \in I} V_{i}-\beta \cdot \sum_{i \in I} \sum_{j \in J} X_{i, j}+\gamma \cdot F
$$

Donde $\alpha, \beta$ y $\gamma$ son constantes que se pueden editar para dar más o menos prioridad en la función de coste a los términos de igualdad en los carros en un mismo proceso de esterilizado, llenado de los autoclaves en los procesos y tiempo final de procesamiento. Esto indica que se obtiene un problema multi-objetivo que debe ser evaludado por los operarios dependiendo del estado actual de la planta, v.gr. se puede disminuir $\alpha$ a fin de permitir la esterilización de carros con procesamientos distintos en el mismo autoclave para evitar que algún carros no sea esterilizado en el margen máximo permitido cuando la exigencia de la línea es muy alta, aumentado $\gamma$ para asegurar que se puede abastecer con suficiente rapidez a las líneas de empaquetado posteriores.

\section{RESULTADOS}

Para la implementación de la formulación matemática y posterior resolución se ha utilizado 


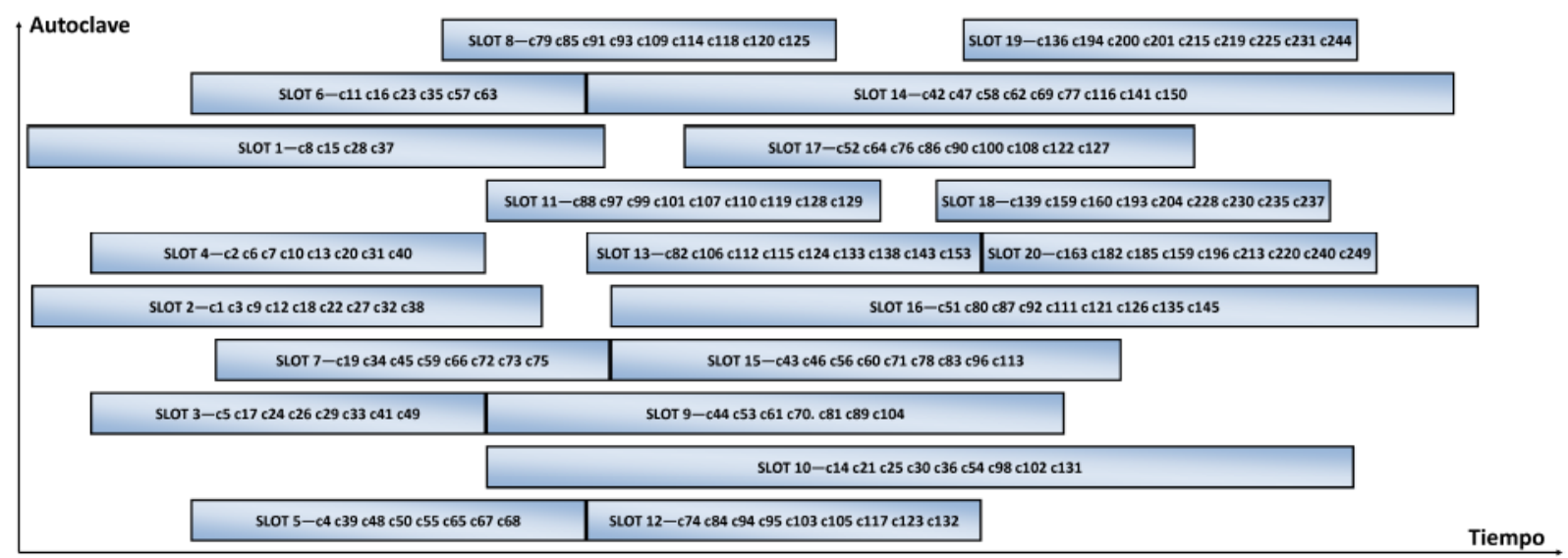

Figura 2: Asignación de autoclaves priorizando similitud entre carros por lote

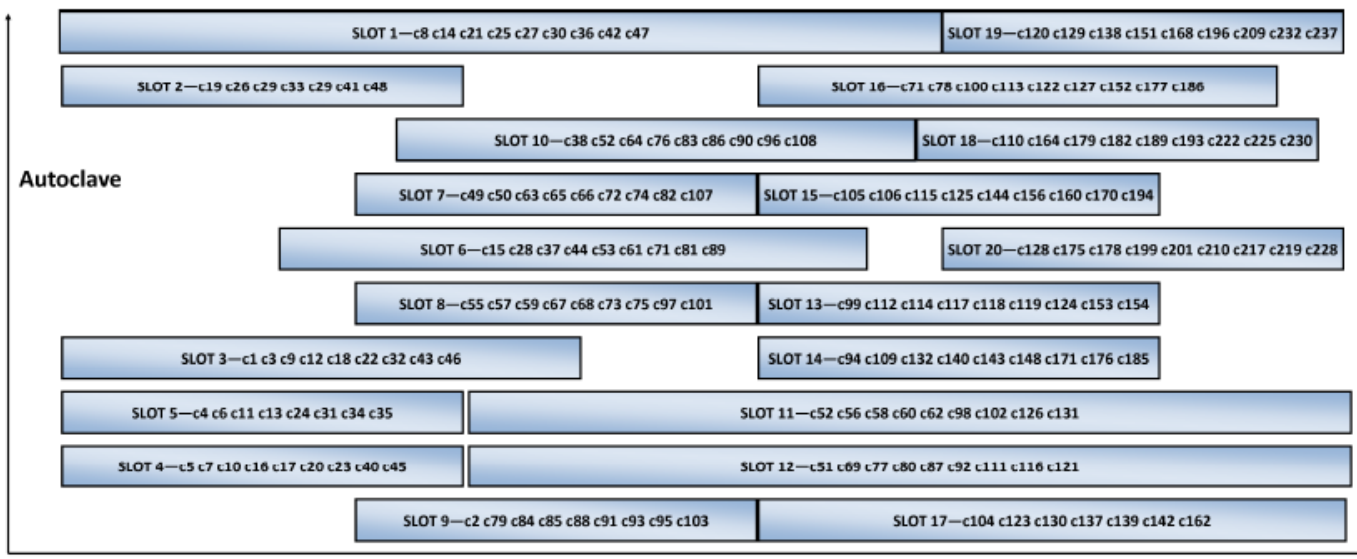

Tiempo

Figura 3: Asignación de autoclaves priorizando acabar en el menor tiempo posible

el software GAMS, en la versión 25.1.1, corriendo sobre Windows 10 Pro. Dado que el problema se ha formulado expresamente como un problema mixto-entero lineal se ha empleado el algoritmo de resolución Cplex de IBM, en la versión 12.8.0.0. El hardware empleado es un Intel ${ }^{\circledR} \mathrm{Core}^{\mathrm{TM}} \mathrm{i} 7-4510 \mathrm{U}$ con 16 GB de memoria RAM.

Para las pruebas se han usado datos reales de planta, por lo que los tiempos de las gráficas no se exponen por motivos de confidencialidad. Se han distribuido un total de 250 carros, entre 10 autoclaves, teniendo que realizar 20 procesos de esterilización. Para visualizar correctamente los distintos resultados obtenidos se muestran dos ejemplos claramente diferenciables: el primero, priorizando la similitud entre carros frente al tiempo de finalización de procesamiento global; y el segundo el opuesto, minimizar el tiempo global final incurriendo en la sobreesterilización de algunos carros. Para ambos casos el tiempo de cómputo es similar, en torno a los diez minutos, siendo un poco más rápida la resolución en el primer caso. Para los dos ejemplos se ha establecido un horizonte de predicción de hora y media, esto es que se debe asegurar que se esterilizan todos los carros que se hayan liberado antes de ese momento.

En las figuras 2 y 3 se han representado mediante diagramas de Gantt las soluciones obtenidas. El eje horizontal representa la evolución temporal, mientras que el eje vertical representa el autoclave al que se ha asignado cada conjunto de carros, etiquetado como "slot"por brevedad. Además, dentro de los conjuntos se indican los carros que pertenecen al mismo etiquetados como $\mathrm{cD}$, siendo $\mathrm{D}$ un dígito desde 1 hasta 250 (el número total de carros que se tienen en cuenta en los ejemplos). En los dos casos se podrían esterilizar un máximo de 180 carros, sin embargo en ninguno de ellos se llega hasta este límite; como era de esperar, cuando no se permiten mezclar productos distintos el número total de carros esterilizados es menor que si se pretende terminar pronto, 166 frente a 175 , ya que también se está pesando maximizar el número de carros a esterilizar.

También puede observarse cómo en la figura 3 los trabajos están más repartidos entre los equipos, y cómo tareas de procesamiento más largas están más llenas que las mismas tareas en la figura 2. 
Otro punto interesante es la elección de carros: los carros que no han llegado antes del horizonte de predicción establecido pueden quedar a la espera de la siguiente ejecución del problema de optimización, por lo que la elección de los mismos depende de sus características, ya que pueden ser más adecuados para un caso que para otro.

\section{CONCLUSIONES Y TRABAJO FUTURO}

Se ha obtenido una herramienta práctica para la resolución en tiempo real de la distribución de cargas entre distintos equipos con capacidades similares, asegurando restricciones temporales de inicio de los procesos. Esto reducirá los tiempos necesarios para el reparto de los lotes, y evitará que haya carros que no cumplean la restricción de margen temporal antes de ser esterilizados, cosa que podría ocurrir en un reparto manual. De esta forma se evita el almacenamiento de estos carros hasta que se realicen los controles de calidad necesarios, reduciendo por lo tanto retrasos en los tiempos de entrega.

Actualmente se están incluyendo los perfiles de consumos de vapor de cada proceso de esterilizado, lo cual permitirá añadir restricciones en el uso de recursos compartidos y la minimización de este consumo como otro término en la función de coste. Este problema puede conllevar la conversión del problema en no lineal, por lo que su aplicación final puede llegar a no ser factible debido a los tiempos de cálculo, por lo que se están estudiando formas de mantener la linealidad.

\section{Agradecimientos}

Este trabajo ha sido financiado por el programa de innovación y desarrollo de la Unión Europea Horizonte 2020, proyecto CoPro (contrato $\mathrm{n}^{\mathrm{O}} 723575$ ) y por el Gobierno de España con fondos MINECO /FEDER (DPI2015-70975-P).

\section{English summary}

\section{OPERATOR SUPPORT IN OP- TIMAL LOAD-ADLLOCATION AMONG EQUIVALENT EQUIP- MENT}

\author{
Abstract \\ This work focuses on the scheduling in a \\ semi-continuous production line of a food \\ plant, more specifically in the distribution \\ of load between similar equipment. Given \\ the special characteristics of the treated
}

product, intended for human consumption, there are harder time and temperature restrictions than in other types of industry.

Unlike the usual planning tools that are used in the overall production planning of the factory, the study presents a tool aiming a real-time application for the operators of a specific section of the production lines: the sterilization, usual bottleneck.

Keywords: Scheduling, RTO, autoclave.

\section{Referencias}

[1] Kopanos, G. M., Puigjaner, L., y Maravelias, C. T. (2010). Production planning and scheduling of parallel continuous processes with product families. Industrial $\& 8$ engineering chemistry research, 50(3), 1369-1378.

[2] Palacín, C. G., Pitarch, J. L., Jasch, C., Méndez, C. A., y de Prada, C. (2017). Robust Integrated Production-Maintenance Scheduling for an Evaporation Network. Computers \& Chemical Engineering.

[3] Wari, E., y Zhu, W. (2016). A survey on metaheuristics for optimization in food manufacturing industry. Applied Soft Computing, $46,328-343$.

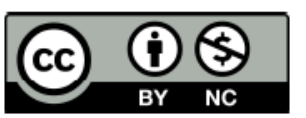

(c) 2018 by the authors. Submitted for possible open access publication under the terms and conditions of the Creative Commons Attribution CC-BY-NC 3.0 license (http://creativecommons.org/licenses/by-nc/3.0/). 